\title{
Tunable Majorana corner modes in noncentrosymmetric superconductors: Tunneling spectroscopy and edge imperfections
}

\author{
S. Ikegaya, ${ }^{1}$ W. B. Rui, ${ }^{2}$ D. Manske, ${ }^{1}$ and Andreas P. Schnyder ${ }^{1}$ \\ ${ }^{1}$ Max-Planck-Institut für Festkörperforschung, Heisenbergstrasse 1, D-70569 Stuttgart, Germany \\ ${ }^{2}$ Department of Physics and HKU-UCAS Joint Institute for Theoretical and Computational Physics at Hong Kong, \\ The University of Hong Kong, Pokfulam Road, Hong Kong, China
}

(Received 1 December 2020; revised 15 February 2021; accepted 26 February 2021; published 2 April 2021)

\begin{abstract}
Majorana corner modes appearing in two-dimensional second-order topological superconductors have great potential applications for fault-tolerant topological quantum computations. We demonstrate that in the presence of an in-plane magnetic field, two-dimensional $(s+p)$-wave superconductors host Majorana corner modes, whose location can be manipulated by the direction of the magnetic field. In addition, we discuss the effects of edge imperfections on the Majorana corner modes. We describe how different edge shapes and edge disorder affect the number and controllability of the Majorana corner modes, which is of relevance for the implementation of topological quantum computations. We also discuss tunneling spectroscopy in the presence of the Majorana corner modes, where a lead wire is attached to the corner of the noncentrosymmetric superconductor. The zero-bias differential conductance shows a distinct periodicity with respect to the direction of the magnetic field, which demonstrates the excellent controllability of the Majorana corner modes in this setup. Our results lay the theoretical groundwork for observing and tuning Majorana corner modes in experiments on $(s+p)$-wave superconductors.
\end{abstract}

DOI: 10.1103/PhysRevResearch.3.023007

\section{INTRODUCTION}

A central subject in the physics of topological superconductivity [1-4] is the realization of fault-tolerant topological quantum computations [5-9] using Majorana zero modes [10-12]. So far, the existence of the Majorana zero modes has been experimentally demonstrated in various systems such as semiconductor-superconductor hybrids [13-27], magnetic atom chains on superconductors [28-31], superconductors in coexistence with nontrivial spin textures [32,33], and superconducting topological insulators [34-39]. However, to achieve the topological quantum computations, we have to manipulate the position of the Majorana zero modes for performing braiding operations, which is still a challenging task at the current stage.

Recently, a fresh route for realizing topological quantum computations has been discussed in the context of higherorder topological superconductivity [40-65]: An $n$ th-order topological superconductor in $d$ dimensions can host Majorana zero modes in $d-n$ dimensions for $n \geqslant 2$, whereas conventional topological superconductors correspond to the case with $n=1$. Although the study of higher-order topological superconductors is still in its infancy, there have already been several theoretical predictions for two-dimensional

Published by the American Physical Society under the terms of the Creative Commons Attribution 4.0 International license. Further distribution of this work must maintain attribution to the author(s) and the published article's title, journal citation, and DOI. Open access publication funded by the Max Planck Society. second-order topological superconductors in which the position of emergent Majorana corner modes (MCMs) can be controlled by varying experimentally tunable parameters [58-65]. Moreover, braiding operations using the advantages of such tunable MCMs have been demonstrated theoretically [63-65]. Thus, this recent development in the physics of tunable MCMs shows great promise for the realization of fault-tolerant topological quantum computations.

A fundamental strategy for obtaining tunable MCMs is the application of magnetic fields to time-reversal-invariant topological superconductors, which host helical Majorana edge states $[43,44,58]$. On the basis of this strategy, the presence of tunable MCMs has been demonstrated in Rashba bilayers coupled to superconductors [60], semiconductor-iron-basedsuperconductor hybrids [59], and spin-triplet helical $p$-wave superconductors [59,64]. Even so, since these proposals have not yet been implemented experimentally, it is important to continue to propose alternative models hosting tunable MCMs. Thus, as the first objective of this paper, we demonstrate that a two-dimensional noncentrosymmetric $(s+p)$-wave superconductor [66-72] can harbor the tunable MCMs by applying in-plane magnetic fields. We mention here that, according to a range of experiments, such as upper critical field measurements [73], Knight-shift measurements [74], and others [75-78], as well as supporting theories [79-82], the noncentrosymmetric compound $\mathrm{CePt}_{3} \mathrm{Si}$ is the most promising candidate for the $(s+p)$-wave superconductor.

Moreover, while most previous studies on tunable MCMs consider square superconducting islands with clean edges, experimental implementations will most certainly deviate from such an ideal geometry. Thus, as the second objective of 
this paper, we study the effects of edge imperfections on the MCMs. For a square geometry with clean edges, we can find two stable Majorana zero modes. However, for more complicated edge configurations, the number of MCMs becomes more than two and depends on the applied magnetic field direction. In this case, we can no longer find a stable pair of Majorana zero modes that can make a full circle around the system, which complicates the braiding process. Thus, the edge configuration is an important factor for accomplishing the topological quantum computations in experiments.

At present, specific theoretical proposals for detecting the tunability of the MCMs are lacking. Thus, as the third objective of this paper, we study the tunneling spectroscopy in the presence of the MCMs, where the normal lead wire is attached to the vicinity of the corner of the noncentrosymmetric superconductor. We demonstrate that the zero-bias conductance becomes a periodic function with respect to the direction of the applied magnetic field. This characteristic periodicity is understood by the fact that the zero-bias conductance is enhanced only when the MCM exits at the corner connected with the lead wire. As a result, we propose a smoking-gun experiment for the detection of controllable MCMs.

The organization of this paper is as follows. In Sec. II we first derive an effective edge theory describing the emergence of tunable MCMs in a noncentrosymmetric superconductor with in-plane magnetic fields. Then we confirm the validity of the edge theory by employing numerical simulations on a two-dimensional tight-binding model. In Sec. III we study the effects of edge imperfections on the tunable MCMs. Specifically, we calculate the local density of states (LDOS) for evaluating the stability and controllability of MCMs in nonsquare superconducting islands. In Sec. IV the differential conductance of normal-lead-wirenoncentrosymmetric-superconductor hybrids is calculated by using lattice Green's function techniques. We demonstrate that the zero-bias conductance shows the periodicity as a function of the direction of the applied magnetic field, which serves as an identifying characteristic of the controllable MCMs. A summary and our conclusions are given in Sec. V.

\section{EDGE THEORY AND TUNABLE MAJORANA CORNER MODES}

\section{A. Model}

Let us consider a two-dimensional noncentrosymmetric $(s+p)$-wave superconductor in the presence of an in-plane magnetic field. We describe the present superconductor by a minimal Bogoliubov-de Gennes (BdG) Hamiltonian [79-81]

$$
\check{H}(\boldsymbol{k})=\check{H}_{\mathrm{ncs}}(\boldsymbol{k})+\check{H}_{Z},
$$

with

$$
\begin{gathered}
\check{H}_{\mathrm{ncs}}(\boldsymbol{k})=\left[\begin{array}{cc}
\hat{h}(\boldsymbol{k}) & \hat{\Delta}(\boldsymbol{k}) \\
-\hat{\Delta}^{*}(-\boldsymbol{k}) & -\hat{h}^{*}(-\boldsymbol{k})
\end{array}\right], \\
\hat{h}(\boldsymbol{k})=\xi(\boldsymbol{k}) \hat{\sigma}_{0}+\lambda \boldsymbol{g}(\boldsymbol{k}) \cdot \hat{\boldsymbol{\sigma}}, \\
\hat{\Delta}(\boldsymbol{k})=\left[\Delta_{s}+\boldsymbol{d}(\boldsymbol{k}) \cdot \hat{\boldsymbol{\sigma}}\right]\left(i \hat{\sigma}_{2}\right), \\
\xi(\boldsymbol{k})=\frac{\hbar^{2} k^{2}}{2 m}-\mu, \quad \boldsymbol{d}(\boldsymbol{k})=\Delta_{t} \frac{\boldsymbol{g}(\boldsymbol{k})}{k_{F}},
\end{gathered}
$$

and

$$
\check{H}_{Z}=\left[\begin{array}{cc}
\boldsymbol{V}_{Z} \cdot \hat{\boldsymbol{\sigma}} & 0 \\
0 & -\left\{\boldsymbol{V}_{Z} \cdot \hat{\boldsymbol{\sigma}}\right\}^{*}
\end{array}\right],
$$

where $m$ is the effective mass of an electron, $\mu$ denotes the chemical potential, $k_{F}=\sqrt{2 m \mu} / \hbar$ represents the Fermi wave number, and $k=\sqrt{k_{x}^{2}+k_{y}^{2}}$. The strength of Rashba spin-orbit coupling, which is due to an intrinsically broken inversion symmetry along the $z$ axis of the material [75], is given by $\lambda$ with $\boldsymbol{g}(\boldsymbol{k})=\left(k_{y},-k_{x}, 0\right)$. The pair potential $\hat{\Delta}(\boldsymbol{k})$ contains both a spin-singlet $s$-wave component $\Delta_{s}$ and a spin-triplet $p$-wave component $\boldsymbol{d}(\boldsymbol{k})$ satisfying $\boldsymbol{d}(\boldsymbol{k}) \| \boldsymbol{g}(\boldsymbol{k})$ [79]. The Zeeman potential induced by the externally applied in-plane magnetic field is described by $\boldsymbol{V}_{Z}=\left(V_{Z} \cos \theta_{Z}, V_{Z} \sin \theta_{Z}, 0\right)$, with $\theta_{Z}$ representing the angle of the magnetic field measured from the $x$ direction. In what follows, without loss of generality, we assume $\lambda, \Delta_{s}, \Delta_{t}, V_{Z} \geqslant 0$. The Pauli matrices in spin space are given by $\hat{\sigma}=\left(\hat{\sigma}_{x}, \hat{\sigma}_{y}, \hat{\sigma}_{z}\right)$ and the $2 \times 2$ unit matrix is denoted by $\sigma_{0}$.

We briefly discuss the topological property of the pure noncentrosymmetric $(s+p)$-wave superconductor described by $\breve{H}_{\text {ncs }}(\boldsymbol{k})$. The positive eigenenergies of $\breve{H}_{\mathrm{ncs}}(\boldsymbol{k})$ are given by

$$
E_{\eta}^{\mathrm{ncs}}(\boldsymbol{k})=\sqrt{\{\xi(\boldsymbol{k})+\eta \lambda k\}^{2}+\left\{\Delta_{t}\left(k / k_{F}\right)+\eta \Delta_{s}\right\}^{2}},
$$

with $\eta= \pm$. While the spectrum of $E_{+}^{\text {ncs }}(\boldsymbol{k})$ has a finite superconducting gap irrespective of the parameters, the superconducting gap in $E_{-}^{\text {ncs }}(\boldsymbol{k})$ vanishes when

$$
\Delta_{s}=\Delta_{c},
$$

with

$$
\Delta_{c}=\Delta_{t}\left[\sqrt{1+\left(\frac{\lambda k_{F}}{2 \mu}\right)^{2}}+\frac{\lambda k_{F}}{2 \mu}\right] .
$$

For $\Delta_{s}>\Delta_{c}\left(\Delta_{s}<\Delta_{c}\right)$, the BdG Hamiltonian $\check{H}_{\text {ncs }}(\boldsymbol{k})$ can be deformed into the BdG Hamiltonian of a pure spin-singlet $s$-wave (pure spin-triplet helical $p$-wave) superconductor without any gap closing by decreasing $\lambda$ and $\Delta_{t}\left(\Delta_{s}\right)$ adiabatically. The spin-triplet helical $p$-wave superconductor is well known as a time-reversal-invariant topological superconductor characterized by a $\mathbb{Z}_{2}$ topological invariant $[83,84]$, whereas the spin-singlet superconductor is topologically trivial. Since the topological invariant never changes without gap closing, the noncentrosymmetric $(s+p)$-wave superconductor with $\Delta_{s}<\Delta_{c}$ is topologically equivalent to the spin-triplet helical $p$-wave superconductor and host the topologically protected helical Majorana edge states [68-72]. In what follows, we focus only on the noncentrosymmetric $(s+p)$-wave superconductor in the topologically nontrivial phase (i.e., $\Delta_{s}<$ $\left.\Delta_{c}\right)$.

Then we discuss the effects of the Zeeman potential against the bulk energy spectrum. Within first-order perturbation theory with respect to $V_{Z}$, the energy spectrum is given by

$$
E_{\eta}(\boldsymbol{k})=E_{\eta}^{\mathrm{ncs}}(\boldsymbol{k})+\eta V_{Z} \sin \left(\theta_{\boldsymbol{k}}-\theta_{Z}\right),
$$

with $\theta_{\boldsymbol{k}}=\arctan k_{y} / k_{x}$. Although the Zeeman potential causes a nonmonotonic energy shift, it is clear that the Zeeman potential suppresses the superconducting gap size, and a large 


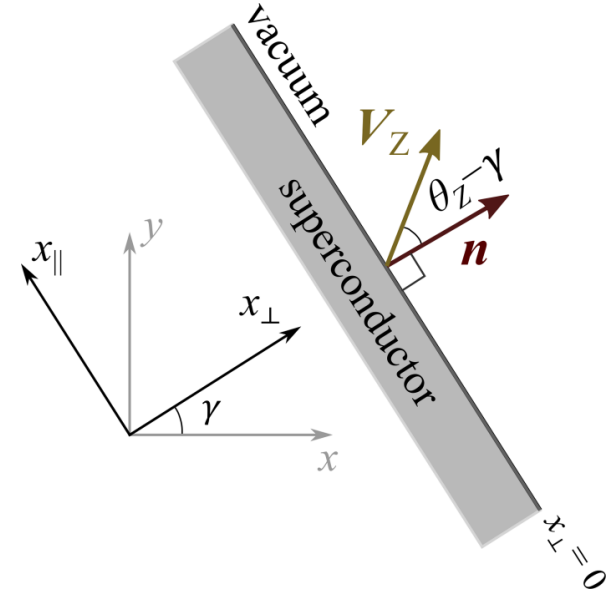

FIG. 1. Edge of the superconductor and relevant spatial coordinates.

enough Zeeman potential may bring the system into a gapless superconducting state similar to the case of the pure helical $p$ wave superconductor discussed in Ref. [85]. Thus, throughout this paper, we only assume weak enough Zeeman potentials such that the bulk superconducting gap remains finite and large enough. Although the superconducting gap remains finite, the noncentrosymmetric $(s+p)$-wave superconductor is no longer characterized by the $\mathbb{Z}_{2}$ topological invariant due to broken time-reversal symmetry. Nevertheless, in the following, we demonstrate that the noncentrosymmetric $(s+$ $p$ )-wave superconductor under the in-plane magnetic field becomes a second-order topological superconductor hosting MCMs.

\section{B. Effective edge Hamiltonian}

In order to discuss the emergence of MCSs in the present system intuitively, we derive an edge theory, similar to the ones discussed in Refs. [46,48,59,60]. We assume here that the present superconductor has an edge perpendicular to $\boldsymbol{n}=$ $(\cos \gamma, \sin \gamma, 0)$, where the spatial coordinate along the direction perpendicular (parallel) to the edge is represented by $x_{\perp}\left(x_{\|}\right)$and the superconductor occupies the entire half space $x_{\perp} \leqslant 0$ (see Fig. 1). In addition, we apply periodic boundary conditions in the direction parallel to the edge (i.e., the direction along the $x_{\|}$axis). To obtain an effective edge Hamiltonian, we rewrite the BdG Hamiltonian in momentum space as

$$
\begin{gathered}
\check{H}(\boldsymbol{k})=\check{H}_{0}(\boldsymbol{k})+\check{H}_{\|}(\boldsymbol{k})+\check{H}_{Z}, \\
\check{H}_{0}(\boldsymbol{k})=\left[\begin{array}{cc}
\hat{h}_{0}(\boldsymbol{k}) & \hat{\Delta}_{0}(\boldsymbol{k}) \\
-\hat{\Delta}_{0}^{*}(-\boldsymbol{k}) & -\hat{h}_{0}^{*}(-\boldsymbol{k})
\end{array}\right], \\
\hat{h}_{0}(\boldsymbol{k})=\xi(\boldsymbol{k})\left(\frac{\hbar^{2} k^{2}}{2 m}-\mu\right) \hat{\sigma}_{0}+\lambda k_{\perp} \hat{\sigma}_{\|}, \\
\hat{\Delta}_{0}(\boldsymbol{k})=\left[\begin{array}{cc}
\Delta_{S}+\frac{\Delta_{t}}{k_{F}} k_{\perp} \hat{\sigma}_{\|}
\end{array}\right]\left(i \hat{\sigma}_{2}\right), \\
\check{H}_{\|}(\boldsymbol{k})=\left[\begin{array}{cc}
\hat{\lambda}_{\|}\left(k_{\|}\right) & \hat{\Delta}_{\|}\left(k_{\|}\right) \\
-\hat{\Delta}_{\|}^{*}\left(-k_{\|}\right) & -\hat{\lambda}_{\|}^{*}\left(-k_{\|}\right)
\end{array}\right],
\end{gathered}
$$

$$
\begin{aligned}
& \hat{\lambda}_{\|}\left(k_{\|}\right)=\lambda k_{\|} \hat{\sigma}_{\perp}, \quad \hat{\Delta}_{\|}\left(k_{\|}\right)=\frac{\Delta_{t}}{k_{F}} k_{\|} \hat{\sigma}_{\perp}\left(i \hat{\sigma}_{2}\right), \\
& \check{H}_{Z}=\left[\begin{array}{cc}
V_{\perp} \hat{\sigma}_{\perp}+V_{\|} \hat{\sigma}_{\|} & 0 \\
0 & -\left\{V_{\perp} \hat{\sigma}_{\perp}+V_{\|} \hat{\sigma}_{\|}\right\}^{*}
\end{array}\right],
\end{aligned}
$$

with

$$
\begin{gathered}
k_{\perp}=k_{x} \cos \gamma+k_{y} \sin \gamma, \\
k_{\|}=-k_{x} \sin \gamma+k_{y} \cos \gamma, \\
\hat{\sigma}_{\perp}=\hat{\sigma}_{x} \cos \gamma+\hat{\sigma}_{y} \sin \gamma, \\
\hat{\sigma}_{\|}=-\hat{\sigma}_{x} \sin \gamma+\hat{\sigma}_{y} \cos \gamma, \\
V_{\perp}=V_{Z} \cos \left(\theta_{Z}-\gamma\right), \quad V_{\|}=V_{Z} \sin \left(\theta_{Z}-\gamma\right),
\end{gathered}
$$

where $k_{\perp}\left(k_{\|}\right)$represent the momentum perpendicular (parallel) to the edge of the superconductor. In what follows, we treat $\check{H}_{\|}(\boldsymbol{k})$ and $\check{H}_{Z}$ as the perturbations. This approximation is justified when $k_{\|} / k_{F} \ll 1$ and when $V_{Z}$ is significantly smaller than the superconducting gap. Moreover, in the following analysis, we assume $\Delta_{s}, \Delta_{t} \ll \lambda k_{F}, \mu$.

For the zeroth-order perturbation, we replace $k_{\perp} \rightarrow-i \partial_{x_{\perp}}$ and find zero-energy states satisfying the equation

$$
\check{H}_{0}\left(-i \partial_{x_{\perp}}, k_{\|}\right) \psi\left(x_{\perp}\right)=0,
$$

with the boundary condition $\psi(0)=\psi(-\infty)=0$. As a result, we find the two solutions

$$
\begin{gathered}
\psi_{+, k_{\|}}\left(x_{\perp}\right)=\frac{1}{2}\left[\begin{array}{c}
e^{-i \gamma / 2} \\
i e^{i \gamma / 2} \\
-e^{i \gamma / 2} \\
-i e^{-i \gamma / 2}
\end{array}\right] \varphi_{+}\left(x_{\perp}\right), \\
\psi_{-, k_{\|}}\left(x_{\perp}\right)=\frac{1}{2}\left[\begin{array}{c}
-i e^{-i \gamma / 2} \\
-e^{i \gamma / 2} \\
i e^{i \gamma / 2} \\
e^{-i \gamma / 2}
\end{array}\right] \varphi_{-}\left(x_{\perp}\right), \\
\varphi_{\zeta}\left(x_{\perp}\right)=2 \sqrt{\kappa} \sin \left(\sqrt{k_{F}^{2}+k_{\lambda}^{2}} x_{\perp}\right) e^{i \zeta k_{\lambda} x_{\perp}} e^{\kappa x_{\perp}}, \\
\kappa=\frac{m \Delta_{t}}{\hbar^{2} k_{F}}, \quad k_{\lambda}=\frac{m \lambda}{\hbar^{2}},
\end{gathered}
$$

with $\zeta= \pm$, where we neglect the insignificant terms of $O\left(\frac{k_{\|}^{2}}{k_{F}^{2}}, \frac{\Delta_{s}}{\mu}, \frac{\Delta_{t}}{\mu}\right)$. The matrix elements of the perturbation terms $\check{H}_{\|}(\boldsymbol{k})+\check{H}_{Z}$ within the first order are calculated as

$$
a_{\zeta, \zeta^{\prime}}=\int_{-\infty}^{0} d x_{\perp} \psi_{\zeta}^{\dagger}\left(x_{\perp}\right)\left\{\check{H}_{\|}(\boldsymbol{k})+\check{H}_{Z}\right\} \psi_{\zeta^{\prime}}\left(x_{\perp}\right) \text {. }
$$

As a result, we obtain an effective edge Hamiltonian

$$
\begin{gathered}
\mathcal{H}^{\prime}=\left[\begin{array}{ll}
a_{+,+} & a_{+,-} \\
a_{-,+} & a_{-,-}
\end{array}\right]=\left[\begin{array}{cc}
V_{\|} & \alpha k_{\|} \\
\alpha^{*} k_{\|} & -V_{\|}
\end{array}\right], \\
\alpha=\frac{\beta\left(\Delta_{t}-i \lambda k_{F}\right)}{k_{F}}, \quad \beta=\frac{\Delta_{t}}{\lambda k_{F}} \frac{4 \mu^{2}+\left(\lambda k_{F}\right)^{2}}{4 \mu^{2}},
\end{gathered}
$$

which is unitarily equivalent to

$$
\mathcal{H}=\left[\begin{array}{cc}
\gamma k_{\|} & V_{\|} \\
V_{\|} & -\gamma k_{\|}
\end{array}\right],
$$



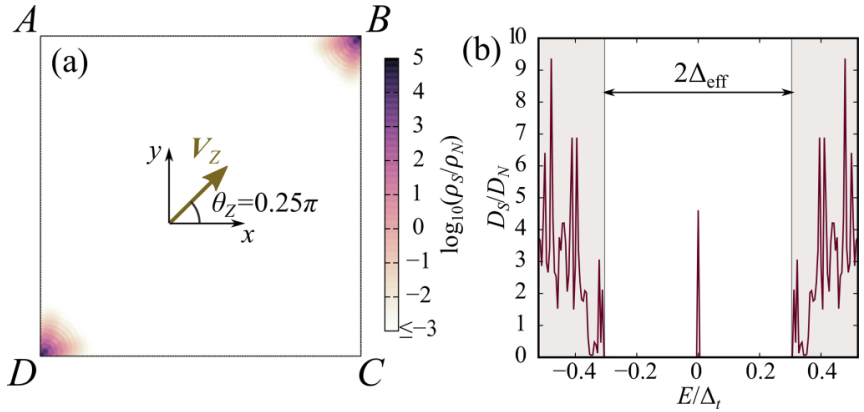

FIG. 2. (a) LDOS at zero energy of the tight-binding model on a square lattice with $201 \times 201$ lattice sites. Specifically, we plot the spatial distribution of $\log _{10}\left(\rho_{S} / \rho_{N}\right)$. The four corners are labeled as $A, B, C$, and $D$, respectively. (b) DOS as a function of energy $E$.

with $\gamma=\beta \sqrt{\Delta_{t}^{2}+\left(\lambda k_{F}\right)^{2}} / k_{F}$. From Eq. (31) we clearly find that the mass term for the linearly dispersive helical edge states is given by $V_{\|}=V_{Z} \sin \left(\theta_{Z}-\gamma\right)$. Importantly, the sign of the mass term is determined only by the relative angle between the Zeeman field $\boldsymbol{V}_{Z}$ and the edge normal vector $\boldsymbol{n}$ :

$$
\begin{array}{ll}
V_{\|}>0 & \text { for } 0<\theta_{Z}-\gamma<\pi, \\
V_{\|}<0 & \text { for }-\pi<\theta_{Z}-\gamma<0, \\
V_{\|}=0 & \text { for } \theta_{Z}-\gamma=0, \pi .
\end{array}
$$

Thus, there is a possibility that two adjacent edges of the system have the mass terms with opposite signs. In such a case, we obtain zero-energy states bounded in the vicinity of the corner (i.e., MCMs) because the mass term has a kink there $[46,48,59]$. In the next section, we confirm the validity of our edge theory by performing numerical calculations of a tight-binding model.

\section{Tunable Majorana corner modes}

We consider here a square superconducting island as shown in Fig. 2(a), where the four corners are labeled as $A$, $B, C$, and $D$, respectively. When we apply the Zeeman field along $\theta_{Z}=0.25 \pi$, according to Eq. (32), the edges $A B$ and $D A$ have negative mass terms, whereas the edges $B C$ and $C D$ have positive mass terms. Therefore, based on the effective edge theory, we can expect MCMs at the corners $B$ and $D$. To confirm the above statement, we numerically calculated the LDOS by using the formula $\rho_{S}(\boldsymbol{r}, E)=-\operatorname{Tr}[\operatorname{Im}\{\breve{G}(\boldsymbol{r}, \boldsymbol{r}, E+$ $i \delta)\}] / \pi$, where $\breve{G}\left(\boldsymbol{r}, \boldsymbol{r}^{\prime}, E\right)$ represents the Green's function and $\operatorname{Tr}$ denotes the trace in spin and Nambu spaces. In addition, $\delta$ is a small imaginary part added to the energy $E$. The Green's function is calculated on a tight-binding model by use of a recursive Green's function technique [86], where we replace $\xi(\boldsymbol{k}) \rightarrow 2 t\left(1-\cos k_{x}\right)+2 t\left(1-\cos k_{y}\right)-\mu, \boldsymbol{g}(\boldsymbol{k}) \rightarrow$ $\left(\sin k_{y},-\sin k_{x}, 0\right)$, and $\Delta_{t} / k_{F} \rightarrow \Delta_{t}$. The explicit expression of the BdG tight-binding Hamiltonian is given in Appendix. In Fig. 2(a) we show the LDOS at zero energy for a $201 \times 201$ square island. We use the parameters $\mu=t, \lambda=0.5 t, \Delta_{t}=$ $0.5 t, \Delta_{s}=0.2 \Delta_{t}, V_{Z}=0.5 \Delta_{t}$, and $\delta=10^{-4} \Delta_{t}$. The LDOS is normalized by $\rho_{N}=\left\langle\rho_{N}(\boldsymbol{r}, E=0)\right\rangle$, where $\rho_{N}(\boldsymbol{r}, E)$ represents the LDOS for the normal state (i.e., $\Delta_{t}=\Delta_{s}=0$ ) and $\langle\cdots\rangle$ means the averaged value with respect to the lattice sites. (a)

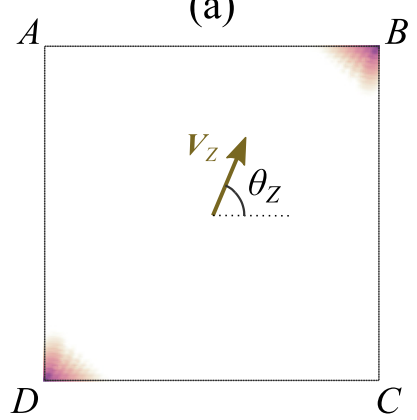

(c)

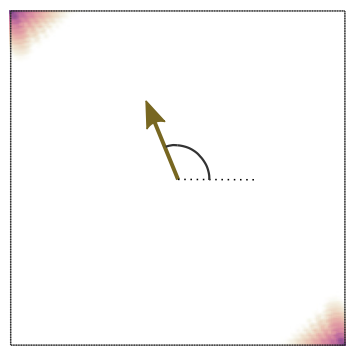

(b)

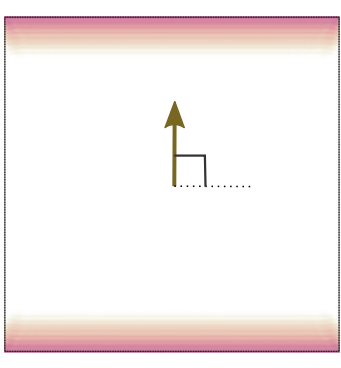

(d)

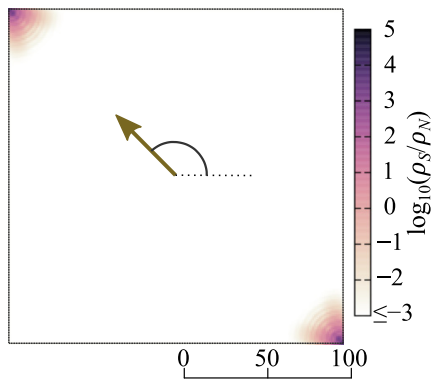

FIG. 3. LDOS at zero energy with (a) $\theta_{Z}=3 \pi / 8$, (b) $\theta_{Z}=\pi / 2$, (c) $\theta_{Z}=5 \pi / 8$, and (d) $\theta_{Z}=3 \pi / 4$.

Moreover, we plot $\log _{10}\left(\rho_{S} / \rho_{N}\right)$ instead of the raw data of $\rho_{S}$. As shown in Fig. 2(a), the LDOS at zero energy has a significant amplitude only in the vicinity of the corners $B$ and $D$. This result confirms the presence of MCMs, as expected. In Fig. 2(b) we show the density of states (DOS) as a function of the energy. The DOS is calculated by $D_{S}(E)=\sum_{\boldsymbol{r} \in S} \rho_{S}(\boldsymbol{r}, E)$, where $\sum_{r \in S}$ represents the summation over the lattice sites in the superconductor island. The results are normalized by the DOS in the normal state $D_{N}=(201 \times 201) \rho_{N}$. As shown in Fig. 2(b), the DOS exhibits a sharp zero-energy peak inside the superconducting gap $\Delta_{\text {eff }}=0.31 \Delta_{t}$. Due to particle-hole symmetry of the superconductor, the two MCMs can depart from zero energy only when they hybridize with each other, thereby becoming conventional electronlike and holelike quasiparticle modes. Therefore, as long as the two MCMs are spatially separated and protected by the superconducting gap $\Delta_{\text {eff }}$, they can remain robust at zero energy.

Next we discuss the controllability of the MCMs. In Fig. 3 we show the LDOS at zero energy with various magnetic field directions, namely, $\theta_{Z}=0.375 \pi$ [Fig. 3(a)], $0.5 \pi$ [Fig. 3(b)], $0.625 \pi / 8$ [Fig. 3(c)], and $0.75 \pi$ [Fig. 3(d)]. When $\theta_{Z}=$ $0.375 \pi$, according to Eq. (32), the kinks of the mass term are located at the corners $B$ and $D$. As a result, although the spatial distributions of the MCMs are slightly modulated from the case with $\theta_{Z}=0.25 \pi$, we can still find them at the corners $B$ and $D$. When $\theta_{Z}=0.5 \pi$, the mass term becomes zero at the entire edges $A B$ and $C D$. As a consequence, zero-energy Majorana edge states appear on the entire edges $A B$ and $C D$. The emergence of Majorana edge states in a time-reversalinvariant topological superconductor under a Zeeman field with a certain direction has been discussed in terms of the Majorana Ising spin [70,87-89] and it has been shown that the energy spectrum of the edge states becomes gapless (strictly speaking, the energy spectrum is discrete due to the finite-size 


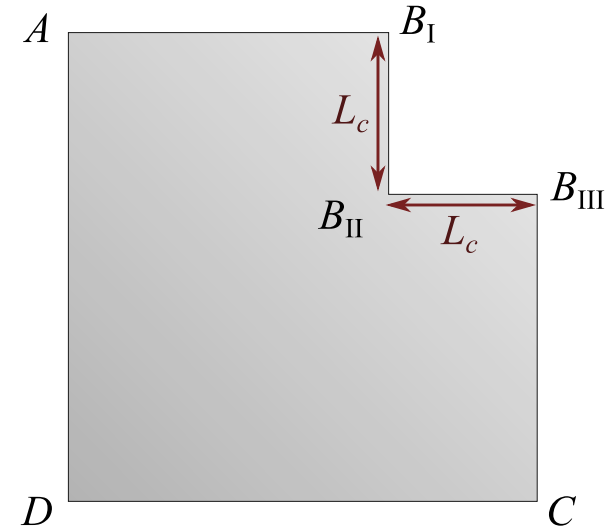

FIG. 4. Nonsquare island obtained by removing an $L_{c} \times L_{c}$ square from the upper right corner of a bigger square.

effect) $[46,70,87-89]$. When $\theta_{Z}$ exceeds $0.5 \pi$, the positions of the mass term kink move to the corner $A$ and $C$. As a consequence, for $\theta_{Z}=0.625 \pi$ and $0.75 \pi$, the MCMs appear at the corners $A$ and $C$. By varying the direction of the Zeeman field from $\theta_{Z}=0.25 \pi$ to $0.75 \pi$, the MCMs originally located at corner $B$ hop to corner $A$. In the same way, the pair of the MCMs can make a full circle around the system by rotating the direction of the Zeeman field by $2 \pi$.

\section{EFFECT OF EDGE IMPERFECTIONS}

In the above, we have demonstrated that the noncentrosymmetric $(s+p)$-wave superconductor can host tunable MCMs. However, in real experiments, the superconducting island generally deviates form the perfect square shape. Thus, in this section we study the effects of different island shapes (Sec. III A) and of edge disorder (Sec. III B).

\section{A. Nonsquare geometry}

As an example of a nonsquare shape, we consider a square island, where a square region of size $L_{c} \times L_{c}$ has been removed from the upper right corner $B$ [46], as shown in Fig. 4. We label the six corners as $A, B_{\mathrm{I}}, B_{\mathrm{II}}, B_{\mathrm{III}}, C$, and $D$. We first consider the MCMs for a field applied along the direction $\theta_{Z}=0.25 \pi$. Under this circumstance, according to Eq. (32), the mass term changes its sign at the four corners $B_{\mathrm{I}}, B_{\mathrm{II}}$, $B_{\mathrm{III}}$, and $D$. In Fig. 5 we show the LDOS at zero energy for an island with the shape of Fig. 4 with $L_{c}=60$ [Fig. 5(a)] and $L_{c}=15$ [Fig. 5(b)]. In accordance with the edge theory, as shown in Fig. 5(a), we find four MCMs for the island with $L_{c}=60$. However, for an island with $L_{c}=15$, there are only two MCMs, where one of them is spread across the corners $B_{\mathrm{I}}, B_{\mathrm{II}}$, and $B_{\mathrm{III}}$. This implies that the three MCMs originally located at $B_{\mathrm{I}}, B_{\mathrm{II}}$ and $B_{\mathrm{III}}$ hybridize with each other, thereby becoming nondegenerate, with two states at small nonzero energy and one state at zero energy, in accordance with particle-hole symmetry. In fact, the edge theory is valid only when the edges are sufficiently longer than the decay length of the MCMs because, to derive the edge theory, we apply the periodic boundary condition in the direction parallel to the edge and do not take into account the length of the edge. Roughly speaking, the decay length of the MCM is evaluated (a)

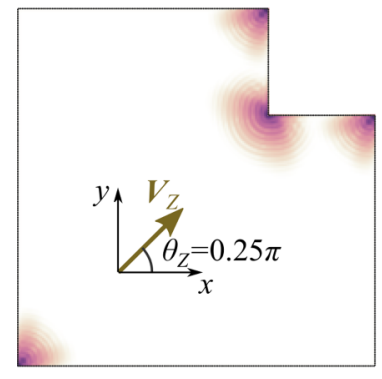

(b)

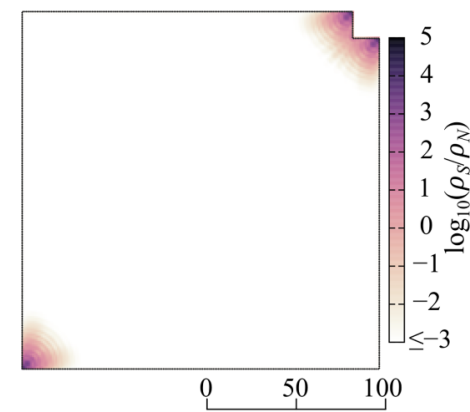

(c)

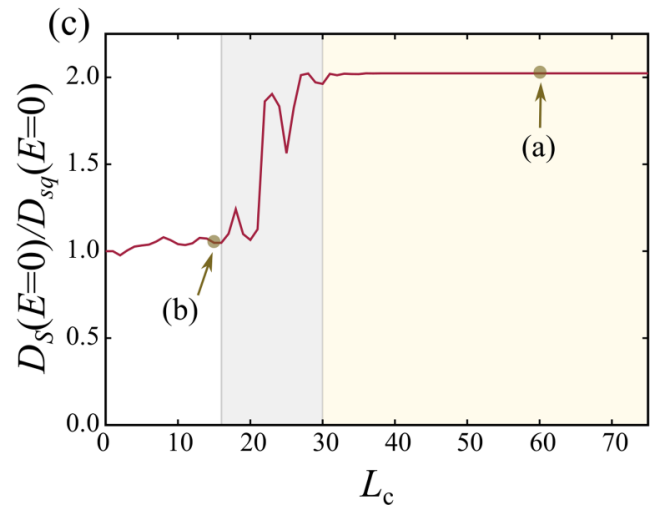

FIG. 5. LDOS at zero energy for the nonsquare island of Fig. 4 with (a) $L_{c}=60$ and (b) $L_{c}=15$. (c) DOS at zero energy as a function of $L_{c}$, where the DOS is normalized by $D_{\mathrm{sq}}(E=0)$ representing the DOS of the ideal square island.

by the inverse of the superconducting gap [90], i.e., $\xi_{\text {eff }} \approx$ $t / \Delta_{\text {eff }}$, where $\xi_{\text {eff }}=6.5$ with the present parameter choices. Thus, when $L_{c}$ exceeds $\xi_{\text {eff }}$ significantly, we obtain the MCMs at the three corners $B_{\mathrm{I}}, B_{\mathrm{II}}$ and $B_{\mathrm{III}}$. In Fig. $5(\mathrm{c})$ we show the DOS at zero energy as a function of $L_{c}$, where the DOS is normalized by $D_{\mathrm{sq}}(E=0)$, representing the zero-energy DOS for the ideal square island, calculated in Fig. 2(b). When $L_{c}$ is large enough (i.e., $L_{c} \gtrsim 30$ ), we find $D_{S} / D_{\mathrm{sq}}=2$. Although the DOS does not count directly the number of states, the relation of $D_{S} / D_{\mathrm{sq}}=2$ implies that the number of MCMs in the present island is twice as much as that in the ideal square island: There are four MCMs as shown in Fig. 4(a). For $L_{c} \lesssim 16$, we find $D_{S} / D_{\mathrm{sq}} \approx 1$, suggesting the presence of two MCMs as shown in Fig. 4(b). In the intermediate region (i.e., $16 \lesssim L_{c} \lesssim 30$ ), the DOS shows nonmonotonic oscillations, which originate from interference effects between the three MCMs, which modify their mutual couplings.

In Fig. 6(a) we show the DOS at zero energy as a function of the angle of the Zeeman field, where we vary $0 \leqslant$ $\theta_{Z} \leqslant 0.625 \pi$ and choose $L_{c}=60$. We find $D_{S} / D_{\mathrm{sq}} \approx 2$ for $0.1 \pi \lesssim \theta_{Z} \lesssim 0.4 \pi$, whereas $D_{S} / D_{\mathrm{sq}} \approx 1$ for $0 \lesssim \theta_{Z} \lesssim 0.06 \pi$ and $0.45 \pi \lesssim \theta_{Z}$. Therefore, the number of MCMs changes by rotating the Zeeman potential. To see more details, in Figs. 6(b)-6(i) we present the LDOS at zero energy for eight different values of $\theta_{Z}$. When $\theta_{Z}=0$, as shown in Fig. 6(b), we find the Majorana edge states at the edge $B_{\mathrm{III}} C$, whereas there is no significant enhancement in the LDOS at the edge $B_{\mathrm{I}} B_{\mathrm{II}}$. When $\theta_{Z}=0.03 \pi$, as shown in Fig. 6(c), an MCM appears at the corner $B_{\mathrm{III}}$, where the wave function is extended 


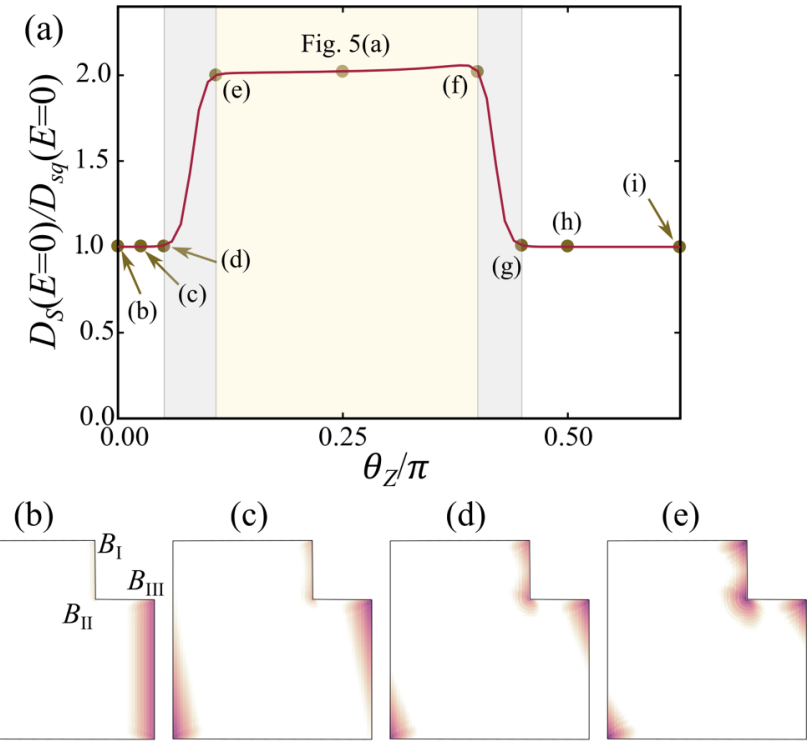

(f)

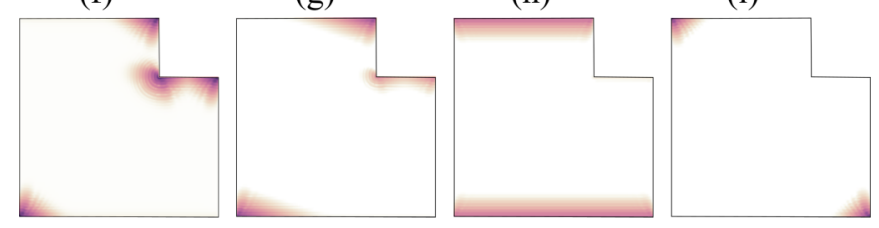

FIG. 6. (a) DOS at zero energy as a function of the angle of the Zeeman field $\theta_{Z}$, where we choose $L_{c}=60$. (b)-(i) LDOS at zero energy for the eight different values of $\theta_{Z}$ indicated in (a): (b) $\theta_{Z}=0$, (c) $\theta_{Z}=0.03 \pi$, (d) $\theta_{Z}=0.06 \pi$, (e) $\theta_{Z}=0.1 \pi$, (f) $\theta_{Z}=0.4 \pi$, (g) $\theta_{Z}=0.45 \pi$, (h) $\theta_{Z}=0.5 \pi$, and (i) $\theta_{Z}=0.625 \pi$.

towards corner $C$. In addition, we find a small enhancement of the LDOS at the edge $B_{\mathrm{I}} B_{\mathrm{II}}$. Strictly speaking, this small enhancement originates not from exact zero-energy states but from the finite-energy states having energy extremely close to zero. We cannot eliminate contributions from such low-energy states because, although the strict definition of the LDOS is given by $\rho_{S}(\boldsymbol{r}, E)=-\lim _{\delta \rightarrow 0+} \operatorname{Tr}[\operatorname{Im}\{\breve{G}(\boldsymbol{r}, \boldsymbol{r}, E+i \delta)\}] / \pi$, the small imaginary part of $\delta$ is always set to be finite in our numerical calculations. As shown in Fig. 6(d), the LDOS at the edge $B_{\mathrm{I}} B_{\mathrm{II}}$ increases by increasing $\theta_{Z}$. When $\theta_{Z}=0.1 \pi$, as shown in Fig. 6(e), we find that the zero-energy LDOS is significantly enhanced in the vicinity of corners $B_{\mathrm{I}}$ and $B_{\mathrm{II}}$. As already shown in Fig. 5(a), with $\theta_{Z}=0.25 \pi$, we obtain three distinct MCMs at the three corners $B_{\mathrm{I}}, B_{\mathrm{II}}$, and $B_{\mathrm{III}}$. As a consequence, by increasing $\theta_{Z}$ from zero to $0.25 \pi$, two additional MCMs are created at corners $B_{\mathrm{I}}$ and $B_{\mathrm{II}}$, while a single MCM stays constantly at corner $B_{\mathrm{II}}$. When $\theta_{Z}=0.4 \pi$, as shown in Fig. 6(e), the wave function of the MCM at corner $B_{\mathrm{III}}$ is extended towards corner $B_{\mathrm{II}}$ such that the MCMs at corners $B_{\mathrm{II}}$ and $B_{\mathrm{III}}$ start to overlap with each other. When $\theta_{Z}=0.45 \pi$, as shown in Fig. 6(e), the LDOS at the edge $B_{\mathrm{II}} B_{\mathrm{III}}$ is strongly suppressed. With $\theta_{Z}=0.5 \pi$, we can no longer find the enhancement of the LDOS at the edge $B_{\mathrm{II}} B_{\mathrm{III}}$, whereas we find a single Majorana edge state at the edge $A B_{\mathrm{I}}$. With $\theta_{Z}=0.625 \pi$, we find only two MCMs at corners $A$ and $C$. Therefore, by increasing $\theta_{Z}$ from $0.25 \pi$ to $0.625 \pi$, the MCMs at corner $B_{\mathrm{II}}$ and that at $B_{\mathrm{III}}$ start to hybridize with
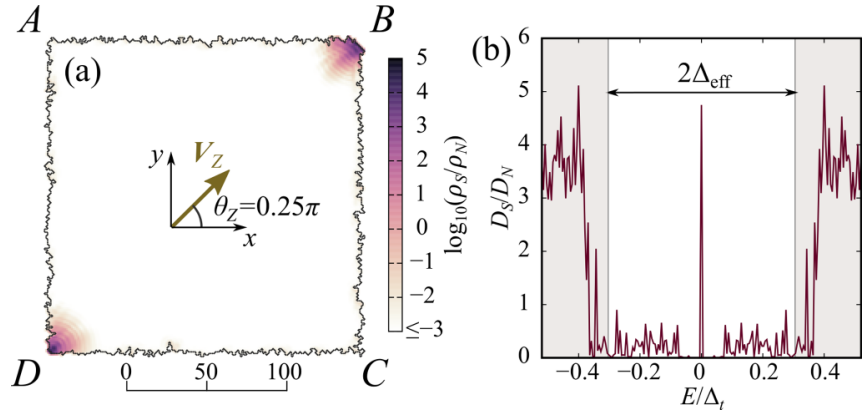

FIG. 7. (a) LDOS at zero energy and (b) DOS as a function of the energy $E$ for the superconducting island with the rough edge $(p, X)=(0.1,20)$.

each other and move away from zero energy. At the same time the single MCM originally located at corner $B_{\mathrm{I}}$ hops to corner $A$, while the single MCM of corner $D$ hops to corner $C$. Importantly, in this process, the MCMs originally appearing at $B_{\mathrm{II}}$ and $B_{\mathrm{III}}$ vanish by hybridizing with each other and never reappear at any other corner. To accomplish the braiding process in topological quantum computations, we must exchange the positions of the two Majorana zero modes forming a pair. In the present edge configuration, however, we can no longer find a stable pair of MCMs that can fully circle the edges of the superconductor, and therefore fail the braiding process. To avoid the emergence of additional and undesired MCMs, as also shown in Fig. 5, we have to eliminate additional corners whose adjacent edges are longer that the decay length of the MCMs.

\section{B. Edge roughness}

Next we consider a square island with rough edges. To described the rough edges, we remove the outermost sites from an ideal square island with probability $p$ and repeat this etching process $X$ times [91,92]. With this process, there is a possibility that small debris separated from the largest island is created. Thus, we also remove such debris. In Figs. 7(a) and 7(b) we show the LDOS at zero energy and the DOS as a function of the energy, respectively. We apply the Zeeman field along $\theta_{Z}=0.25 \pi$ and consider a rough edge with $(p, X)=(0.1,20)$. We find that the zero-energy LDOS in the vicinity of corners $B$ and $D$ is enhanced significantly. In addition, as shown in Fig. 7(b), the DOS retains a steep zero-energy peak structure, whereas we also find a finite DOS inside the effective superconducting gap in the clean limit $\Delta_{\text {eff }}=0.31 \Delta_{t}$. As also discussed in Sec. II C, the MCMs are protected by the particle-hole symmetry as long as they are sufficiently separated spatially. Thus, the MCMs remain robust even in the presence of weak or moderate edge roughness that does not destroy the superconducting gap completely.

In Fig. 8(a) we show the normalized DOS at zero energy as a function of the direction of the Zeeman field. For the solid red line, we use the same superconducting island as in Fig. 7 (a), i.e., $(p, X)=(0.1,20)$. For the dotted black line, we consider an island with weaker roughness $(p, X)=(0.1,5)$. At first, we concentrate on the results with $(p, X)=(0.1,20)$, shown by the solid red line. For $0.1 \pi \lesssim \theta_{Z} \lesssim 0.4 \pi$ and for $0.69 \pi \lesssim \theta_{Z} \lesssim 0.89 \pi$, we find $D_{S} / D_{\mathrm{sq}} \approx 1$, suggesting the 


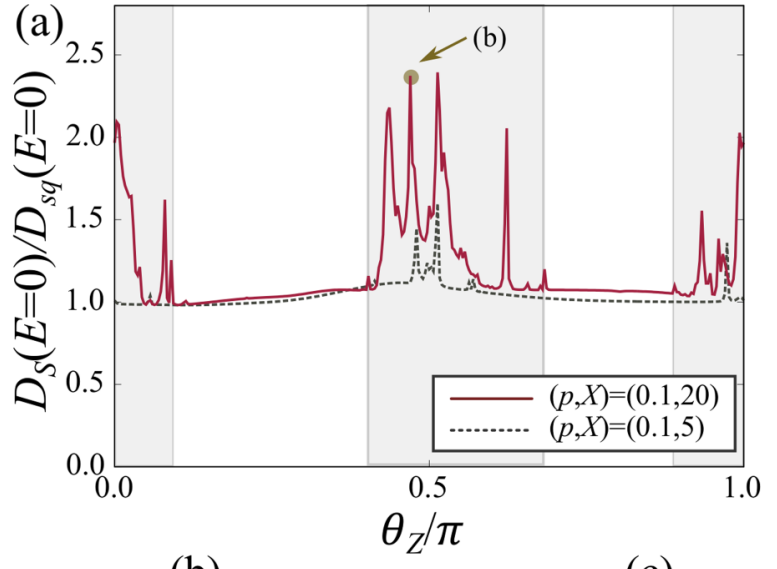

(b)

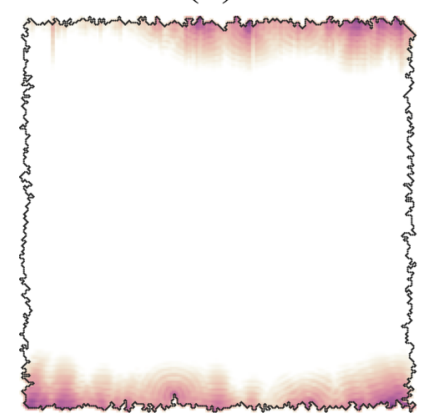

(c)

FIG. 8. (a) DOS at zero energy as a function of the angle of the Zeeman field $\theta_{Z}$. The solid red line denotes the result with the same island as in Fig. 7(a) [i.e., $(p, X)=(0.1,20)$ ] and the dotted black line denotes the result for the island with weaker roughness $(p, X)=$ $(0.1,5)$. (b) and (c) LDOS at zero energy with $\theta_{Z}=0.47 \pi$. In (b) we use the same island as in Fig. 7(a) [i.e., $(p, X)=(0.1,20)]$. In (c), for comparison, we show the result with clean edges.

presence of distinct MCMs. However, in the vicinity of $\theta_{Z}=$ $0,0.5 \pi$, and $\pi$, the DOS shows a nonmonotonic dependence and becomes clearly larger than one, i.e., $D_{S} / D_{\mathrm{sq}}>1$. To see more details, in Fig. 8(b) we show the LDOS at zero energy with $\theta_{Z}=0.47 \pi$, where we use the same superconducting island for calculating the solid red line in Fig. 8(a). For comparison, in Fig. 8(c) we also show the result with the clean edge. With the rough edge, we find that the LDOS is enhanced not only in the vicinity of corners $B$ and $D$ but also around the center of the edge $A B$ and at corner $C$. According to the effective edge theory, when $\theta_{Z}$ is close to $0.5 \pi$, the mass term $V_{\|}=V_{Z} \sin \left(\theta_{Z}-\gamma\right)$, which is the source of the energy gap in the edge spectrum, is suppressed at the edges $A B$ and $C D$. In particular, the mass term of the edges $A B$ and $C D$ vanishes completely at $\theta=0.5 \pi$. The results in Fig. 8 suggest that the already small energy gap is reduced further by the edge roughness such that states with extremely low (but finite) energy appear at the edges $A B$ and $C D$. In the topological quantum computations, the energy gap which energetically separates the MCMs from other finite-energy states plays an essential role in suppressing the decoherence during the braiding operations. Therefore, the edge roughness causing damage to the energy gap in the edge spectrum affects negatively the braiding processes of the MCMs. As shown by the dotted black line in Fig. 8(a), the undesirable enhancement $(s+p)$-wave superconductor

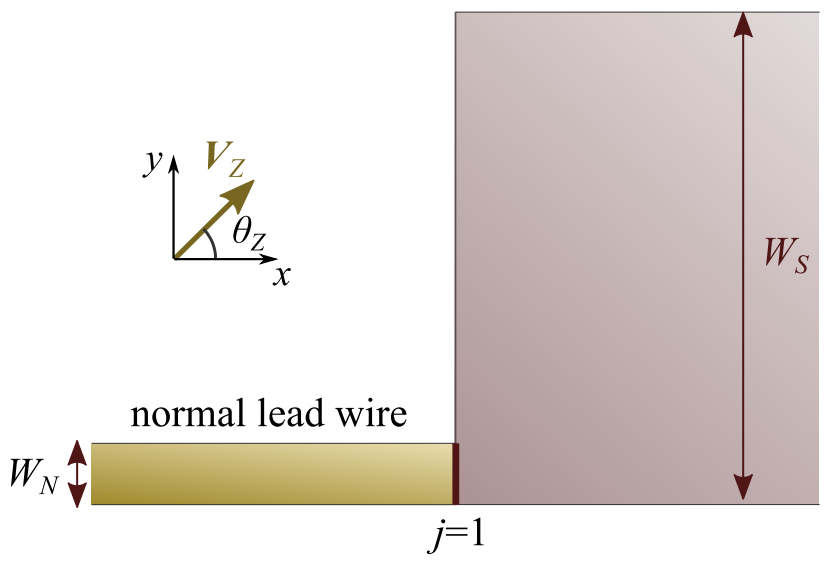

FIG. 9. Schematic image of the normal-lead-wire- $(s+p)$-wave superconductor junction.

in the low-energy DOS is suppressed by decreasing the edge roughness.

In the above, we have shown that, although the MCMs exist robustly, the braiding operation of them may be disturbed by edge imperfections. The simplest countermeasure would be fabricating superconducting islands with clean and smooth edges. Even so, establishing a practical way to to make the braiding process more robust would be a desirable work [63-65].

\section{TUNNELING SPECTROSCOPY}

Finally, we propose an experiment for detecting the tunable MCMs. Let us consider a normal-lead-wire-superconductor junction, where the normal lead wire is attached to a corner of the superconductor as shown in Fig. 9. We describe the present junction with a tight-binding model, where a lattice site is indicated by $\boldsymbol{r}=j \boldsymbol{x}+m \boldsymbol{y}$, with $\boldsymbol{x}(\boldsymbol{y})$ representing the unit vector in the $x(y)$ direction. The superconductor (lead wire) is located at $1 \leqslant j \leqslant \infty(-\infty \leqslant j<1)$ and $1 \leqslant m \leqslant$ $W_{S}\left(1 \leqslant m \leqslant W_{N}\right)$, where $W_{S}\left(W_{N}\right)$ denotes the width of the superconductor (lead wire). The superconducting segment is described by the BdG Hamiltonian used also in the above numerical calculations, and the normal lead wire is described by setting $\Delta_{t}=\Delta_{s}=0$ and $\lambda=0$. The hopping integral between the superconductor and the lead wire (i.e., $j=0$ and $j=1$ ) is given by $t_{\text {int }}$. The explicit form of the BdG Hamiltonian describing the present junction is given in Appendix. In the following calculations, we set the parameters as $\mu=$ $t, \lambda=0.5 t, \Delta_{t}=0.5 t, \Delta_{s}=0.2 \Delta_{t}, V_{Z}=0.5 \Delta_{t}, t_{\text {int }}=0.1 t$, $W_{N}=10$, and $W_{S}=101$. We assume a sufficiently low transparency at the junction interface (i.e., $t_{\text {int }}=0.1 t$ ) such that the bias voltage is mainly dropped at the interface. Under this circumstance, we can calculate the differential conductance at zero temperature by using the Blonder-Tinkham-Klapwijk formula [93-95]

$$
G(e V)=\frac{e^{2}}{h} \sum_{\zeta_{\sigma}, \eta_{\sigma^{\prime}}}\left[\delta_{\zeta_{\sigma}, \eta_{\sigma^{\prime}}}-\left|r_{\zeta_{\sigma}, \eta_{\sigma^{\prime}}}^{e e}\right|^{2}+\left|r_{\zeta_{\sigma}, \eta_{\sigma^{\prime}}}^{h e}\right|^{2}\right]_{E=e V}
$$



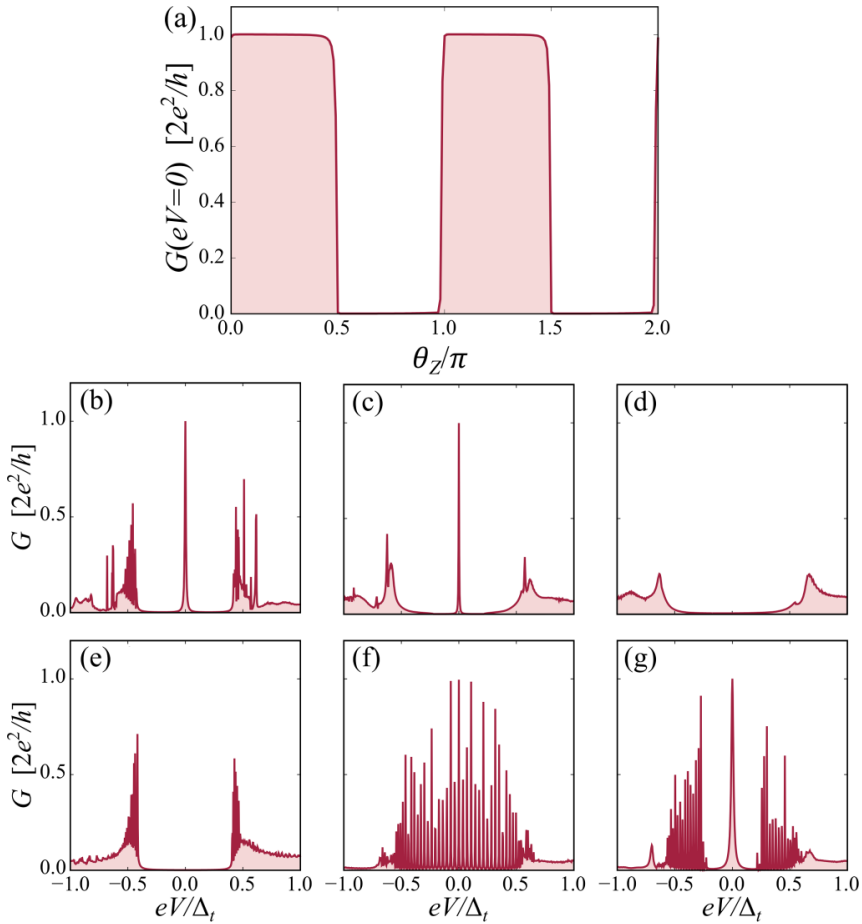

FIG. 10. (a) Zero-bias conductance as a function of the angle of the Zeeman field $\theta_{Z}$. (b)-(g) Differential conductance as a function of the bias voltages for (b) $\theta_{Z}=0.25 \pi$, (c) $\theta_{Z}=0.375 \pi$, (d) $\theta_{Z}=$ $0.5 \pi$, (e) $\theta_{Z}=0.75 \pi$, (f) $\theta_{Z}=\pi$, and (g) $\theta_{Z}=1.125 \pi$.

where $r_{\zeta_{\sigma}, \eta_{\sigma^{\prime}}}^{e e}$ and $r_{\zeta_{\sigma}, \eta_{\sigma^{\prime}}}^{h e}$ denote a normal and an Andreev reflection coefficient at energy $E$, respectively. The index $\zeta_{\sigma}$ $\left(\eta_{\sigma^{\prime}}^{\prime}\right)$ labels an outgoing (incoming) channel with spin $\sigma\left(\sigma^{\prime}\right)$. These reflection coefficients are calculated using recursive Green's function techniques [86,96].

In Fig. 10(a) we show the zero-bias conductance $\mathrm{G}(\mathrm{eV}=$ $0)$ as a function of the angle of the Zeeman field $\theta_{Z}$. We find the conductance plateaus at $2 e^{2} / h$ for $0<\theta_{Z}<0.5 \pi$ and $\pi<\theta_{Z}<1.5 \pi$. For these $\theta_{Z}$, according to the effective edge theory in Sec. II B, the MCM appears at the corner attached to the lead wire. Therefore, it is clear that the conductance plateau is caused by the resonant tunneling through the MCM [97-100]. To see more details, in Figs. 10(b)-10(g) we show the differential conductance as a function of the bias voltage for various $\theta_{Z}$. At $\theta_{Z}=0.25 \pi$, as shown in Fig. 10(b), we find a steep zero-bias conductance peak, where the conductance spectrum for finite energies displays an almost hard-gap structure. For $\theta_{Z}=0.375 \pi$, we still find the clear peak, whereas the superconducting gap edge has now a tail that was absent for $\theta_{Z}=0.25 \pi$. When $\theta_{Z}=0.5 \pi$, the Majorana edge mode appears along the edge perpendicular to the junction interface. Since the Majorana edge mode is not bounded along the junction interface, it cannot cause resonant tunneling [101]. As a result, as shown in Fig. 10(d), the zero-bias conductance peak disappears when $\theta_{Z}=0.5 \pi$. At $\theta_{Z}=0.75 \pi$, there are no zero-energy states in the vicinity of the corner attached to the lead wire. As a consequence, we find a hard gap in the conductance spectrum. When $\theta_{Z}=\pi$, the gapless Majorana edge states appear at the edge along the junction interface.
Here we must note that the energy levels of the Majorana edge states are discretized owing to the finite size of the system. As a consequence, as shown in Fig. 10(f), the conductance spectrum exhibits many spikes for low-bias voltages. When we prepare a sufficiently large superconductor, the energy levels of the Majorana edge states will form a continuum such that the conductance spectra show a broad peak structure instead of a collection of sharp peaks. With $\theta_{Z}=1.125 \pi$, the MCM again appears at the corner attached to the lead wire. As a consequence, as shown in Fig. 10(g), the conductance spectrum exhibits a zero-bias peak, whereas we also find significant enhancement in low-bias voltages. When $\theta_{Z}=1.25 \pi$, the conductance spectrum returns to that in Fig. 10(b).

The periodicity of the zero-bias conductance with respect to the direction of the Zeeman field is smoking gun evidence for the existence of controllable MCMs. In real experiments, finite-temperature effects and other perturbations may modify the details of the conductance spectrum. Moreover, the edge roughness may disturb the stepwise change in the zerobias conductance shown in Fig. 10(a) because, according to Sec. III B, the edge roughness affects significantly the spectrum of the edge states around $\theta=0,0.5 \pi, \pi$, and $1.5 \pi$. Nonetheless, our proposal is still valid for identifying the controllable MCMs because we only need the distinct periodicity in the zero-bias conductance and we do not need any detailed and quantitative information about the conductance spectrum.

\section{CONCLUSION}

We have demonstrated that a two-dimensional noncentrosymmetric $(s+p)$-wave superconductor in the presence of an in-plane magnetic field can host tunable MCMs. We also show that the number of MCMs depends sensitively on the edge shape of the noncentrosymmetric superconductor. We find that with irregular edge shapes there are no stable pairs of MCMs that can fully circle around the edges of the superconductor, a property that is important for braiding. We have also studied the effects of edge roughness on the MCMs and found that the MCMs are in general robust to edge roughness. However, edge roughness suppresses the edge gap and may cause undesirable decoherence effects during the braiding process. As a result, although the MCMs can be found to be insensitive to the edge configurations, the edge imperfections may disturb the realization of topological computations. Therefore, developing practical measures to stabilize the braiding process of tunable MCMs, as well as fabricating clean devices, would be important future tasks for realizing topological quantum computations. In addition, we discuss the tunneling spectroscopy in the presence of the MCMs, where a normal lead wire is attached to a corner of the noncentrosymmetric superconductor. The periodicity in the zero-bias conductance with respect to the magnetic field direction is smoking-gun evidence of the existence of controllable MCMs. The proposed experiment is promising for observing tunable MCMs, because we can expect the periodicity in the zero-bias conductance to be insensitive to the details of the model. In conclusion, we have provided groundwork knowledge for designing specific experiments 
for detecting tunable MCMs, which is an essential step for realizing future topological quantum computations using higher-order topological superconductors.

\section{ACKNOWLEDGMENTS}

This work was supported by the JSPS Core-to-Core program "Oxide Superspin" international network. This research was supported in part by the National Science Foundation through Grant No. NSF PHY-1748958.

\section{APPENDIX: BOGOLIUBOV-DE GENNES HAMILTONIAN IN A TIGHT-BINDING MODEL}

In this Appendix we show the explicit form of the BdG Hamiltonian in the tight-binding model. A lattice site is indicated by $\boldsymbol{r}=j \boldsymbol{x}+m \boldsymbol{y}$, with $\boldsymbol{x}(\boldsymbol{y})$ representing the unit vector in the $x(y)$ direction. We assume that the superconductor is occupied for $1 \leqslant j \leqslant L_{S}$ and $1 \leqslant m \leqslant W_{S}$ and the hard-wall boundary condition is applied in both the $x$ and $y$ directions. The BdG Hamiltonian reads $H=H_{N}+H_{\Delta}$, with

$$
\begin{aligned}
H_{N}= & -t \sum_{\sigma=\uparrow, \downarrow}\left[\sum_{j=1}^{L_{S}-1} \sum_{m=1}^{W_{S}}\left\{c_{\boldsymbol{r}+\boldsymbol{x}, \sigma}^{\dagger} c_{\boldsymbol{r}, \sigma}+c_{\boldsymbol{r}, \sigma}^{\dagger} c_{\boldsymbol{r}+\boldsymbol{x}, \sigma}\right\}+\sum_{j=+1}^{L_{S}} \sum_{m=1}^{W_{S}-1}\left\{c_{\boldsymbol{r}+\boldsymbol{y}, \sigma}^{\dagger} c_{\boldsymbol{r}, \sigma}+c_{\boldsymbol{r}, \sigma}^{\dagger} c_{\boldsymbol{r}+\boldsymbol{y}, \sigma}\right\}\right]+\sum_{j=1}^{L_{S}} \sum_{m=1}^{W_{S}} \sum_{\sigma}(4 t-\mu) c_{\boldsymbol{r}, \sigma}^{\dagger} c_{\boldsymbol{r}, \sigma} \\
& +\frac{i \lambda}{2} \sum_{\sigma, \sigma^{\prime}}\left[\sum_{j=+1}^{L_{S}-1} \sum_{m=1}^{W_{S}}(\hat{\sigma})_{\sigma, \sigma^{\prime}}\left\{c_{\boldsymbol{r}+\boldsymbol{x}, \sigma}^{\dagger} c_{\boldsymbol{r}, \sigma^{\prime}}-c_{\boldsymbol{r}, \sigma}^{\dagger} c_{\boldsymbol{r}+\boldsymbol{x}, \sigma^{\prime}}\right\}-\sum_{j=+1}^{L_{S}} \sum_{m=1}^{W_{S}-1}\left(\hat{\sigma}_{x}\right)_{\sigma, \sigma^{\prime}}\left\{c_{\boldsymbol{r}+\boldsymbol{y}, \sigma}^{\dagger} c_{\boldsymbol{r}, \sigma^{\prime}}-c_{\boldsymbol{r}, \sigma}^{\dagger} c_{\boldsymbol{r}+\boldsymbol{y}, \sigma^{\prime}}\right\}\right] \\
& +\sum_{j=1}^{L_{S}} \sum_{m=1}^{W_{S}} \sum_{\sigma, \sigma^{\prime}}\left(\boldsymbol{V}_{Z} \cdot \hat{\boldsymbol{\sigma}}\right)_{\sigma, \sigma^{\prime}} c_{\boldsymbol{r}, \sigma}^{\dagger} c_{\boldsymbol{r}, \sigma^{\prime}}
\end{aligned}
$$

and

$$
\begin{aligned}
H_{\Delta}= & \frac{i \Delta_{t}}{4} \sum_{\sigma, \sigma^{\prime}}\left[-i \sum_{j=+1}^{L_{S}-1} \sum_{m=1}^{W_{S}} \delta_{\sigma, \sigma^{\prime}}\left\{c_{\boldsymbol{r}+\boldsymbol{x}, \sigma}^{\dagger} c_{\boldsymbol{r}, \sigma^{\prime}}^{\dagger}-c_{\boldsymbol{r}, \sigma}^{\dagger} c_{\boldsymbol{r}+\boldsymbol{x}, \sigma^{\prime}}^{\dagger}\right\}-\sum_{j=+1}^{L_{S}} \sum_{m=1}^{W_{S}-1}\left(\hat{\sigma}_{z}\right)_{\sigma, \sigma^{\prime}}\left\{c_{\boldsymbol{r}+\boldsymbol{y}, \sigma}^{\dagger} c_{\boldsymbol{r}, \sigma^{\prime}}^{\dagger}-c_{\boldsymbol{r}, \sigma}^{\dagger} c_{\boldsymbol{r}+\boldsymbol{y}, \sigma^{\prime}}^{\dagger}\right\}\right] \\
& +\sum_{j=1}^{L_{S}} \sum_{m=1}^{W_{S}} \Delta_{S} c_{\boldsymbol{r}, \uparrow}^{\dagger} c_{\boldsymbol{r}, \downarrow}^{\dagger}+\text { H.c., }
\end{aligned}
$$

where $c_{r, \sigma}^{\dagger}\left(c_{r, \sigma}\right)$ is the creation (annihilation) operator of an electron at $\boldsymbol{r}$ with spin $\sigma=\uparrow, \downarrow, t$ denotes the nearest-neighbor hopping integral, and $\mu$ is the chemical potential. The strength of the Rashba spin-orbit coupling is represented by $\lambda$. The Zeeman potential due to the externally applied in-plane magnetic field is given by $\boldsymbol{V}_{Z}=\left(V_{Z} \cos \theta_{Z}, V_{Z} \sin \theta_{Z}, 0\right)$. The spin-singlet $s$-wave and spin-triplet $p$-wave pair potentials are denoted by $\Delta_{s}$ and $\Delta_{t}$, respectively. The Pauli matrices in spin space are given by $\hat{\sigma}=\left(\hat{\sigma}_{x}, \hat{\sigma}_{y}, \hat{\sigma}_{z}\right)$. The normal lead wire in Fig. 9 is described by $H_{N}$ with re- placing $L_{S} \rightarrow-\infty, W_{S} \rightarrow W_{N}$, and $\lambda \rightarrow 0$. The coupling between the lead wire and the superconductor is described by

$$
H_{\mathrm{int}}=-t_{\mathrm{int}} \sum_{m=1}^{W_{N}} \sum_{\sigma}\left[c_{j=1, m, \sigma}^{\dagger} c_{0, m, \sigma}+c_{0, m, \sigma}^{\dagger} c_{1, m, \sigma}\right],
$$

where $t_{\text {int }}$ denotes the hopping integral at the junction interface.
[1] M. Z. Hasan and C. L. Kane, Colloquium: Topological insulators, Rev. Mod. Phys. 82, 3045 (2010).

[2] X.-L. Qi and S.-C. Zhang, Topological insulators and superconductors, Rev. Mod. Phys. 83, 1057 (2011).

[3] Y. Tanaka, M. Sato, and N. Nagaosa, Symmetry and topology in superconductors-Odd-frequency pairing and edge states, J. Phys. Soc. Jpn. 81, 011013 (2012).

[4] M. Sato and Y. Ando, Topological superconductors: A review, Rep. Prog. Phys. 80, 076501 (2017).

[5] D. A. Ivanov, Non-Abelian Statistics of Half-Quantum Vortices in $p$-Wave Superconductors, Phys. Rev. Lett. 86, 268 (2001).

[6] A. Y. Kitaev, Fault-tolerant quantum computation by anyons, Ann. Phys. (NY) 303, 2 (2003).
[7] C. Nayak, S. H. Simon, A. Stern, M. Freedman, and S. Das Sarma, Non-Abelian anyons and topological quantum computation, Rev. Mod. Phys. 80, 1083 (2008).

[8] J. Alicea, Y. Oreg, G. Refael, F. v. Oppen, and M. P. A. S. Fisher, Non-Abelian statistics and topological quantum information processing in 1D wire networks, Nat. Phys. 7, 412 (2011).

[9] S. Das Sarma, M. Freedman, and C. Nayak, Majorana zero modes and topological quantum computation, npj Quantum Inf. 1, 15001 (2015).

[10] N. Read and D. Green, Paired states of fermions in two dimensions with breaking of parity and time-reversal symmetries and the fractional quantum Hall effect, Phys. Rev. B 61, 10267 (2000). 
[11] A. Y. Kitaev, Unpaired Majorana fermions in quantum wires, Phys. Usp. 44, 131 (2001).

[12] F. Wilczek, Majorana returns, Nat. Phys. 5, 614 (2009).

[13] R. M. Lutchyn, J. D. Sau, and S. Das Sarma, Majorana Fermions and a Topological Phase Transition in Semiconductor-Superconductor Heterostructures, Phys. Rev. Lett. 105, 077001 (2010).

[14] Y. Oreg, G. Refael, and F. von Oppen, Helical Liquids and Majorana Bound States in Quantum Wires, Phys. Rev. Lett. 105, 177002 (2010).

[15] V. Mourik, K. Zuo, S. M. Frolov, S. R. Plissard, E. P. A. M. Bakkers, and L. P. Kouwenhoven, Signatures of Majorana fermions in hybrid superconductor-semiconductor nanowire devices, Science 336, 1003 (2012).

[16] M. T. Deng, C. L. Yu, G. Y. Huang, M. Larsson, P. Caroff, and H. Q. Xu, Anomalous zero-bias conductance peak in a NbInSb nanowire-Nb hybrid device, Nano Lett. 12, 6414 (2012).

[17] H. Zhang, C.-X. Liu, S. Gazibegovic, D. Xu, J. A. Logan, G. Wang, N. van Loo, J. D. S. Bommer, M. W. A. de Moor, D. Car, R. L. M. Op het Veld, P. J. van Veldhoven, S. Koelling, M. A. Verheijen, M. Pendharkar, D. J. Pennachio, B. Shojaei, J. S. Lee, C. J. Palmstrøm, E. P. A. M. Bakkers, S. Das Sarma, and L. P. Kouwenhoven, Quantized Majorana conductance, Nature (London) 556, 74 (2018).

[18] M. Hell, M. Leijnse, and K. Flensberg, Two-Dimensional Platform for Networks of Majorana Bound States, Phys. Rev. Lett. 118, 107701 (2017).

[19] F. Pientka, A. Keselman, E. Berg, A. Yacoby, A. Stern, and B. I. Halperin, Topological Superconductivity in a Planar Josephson Junction, Phys. Rev. X 7, 021032 (2017).

[20] A. Haim and A. Stern, Benefits of Weak Disorder in OneDimensional Topological Superconductors, Phys. Rev. Lett. 122, 126801 (2019).

[21] F. Setiawan, A. Stern, and E. Berg, Topological superconductivity in planar Josephson junctions: Narrowing down to the nanowire limit, Phys. Rev. B 99, 220506(R) (2019).

[22] S. Ikegaya, S. Tamura, D. Manske, and Y. Tanaka, Anomalous proximity effect of planar topological Josephson junctions, Phys. Rev. B 102, 140505(R) (2020).

[23] A. Fornieri, A. M. Whiticar, F. Setiawan, E. Portolés, A. C. C. Drachmann, A. Keselman, S. Gronin, C. Thomas, T. Wang, R. Kallaher, G. C. Gardner, E. Berg, M. J. Manfra, A. Stern, C. M. Marcus, and F. Nichele, Evidence of topological superconductivity in planar Josephson junctions, Nature (London) 569, 89 (2019).

[24] H. Ren, F. Pientka, S. Hart, A. T. Pierce, M. Kosowsky, L. Lunczer, R. Schlereth, B. Scharf, E. M. Hankiewicz, L. W. Molenkamp, B. I. Halperin, and A. Yacoby, Topological superconductivity in a phase-controlled Josephson junction, Nature (London) 569, 93 (2019).

[25] T. Zhou, M. C. Dartiailh, W. Mayer, J. E. Han, A. MatosAbiague, J. Shabani, and I. Žutić, Phase Control of Majorana Bound States in a Topological X Junction, Phys. Rev. Lett. 124, 137001 (2020).

[26] M. C. Dartiailh, W. Mayer, J. Yuan, K. S. Wickramasinghe, A. Matos-Abiague, I. Žutić, and J. Shabani, Phase Signature of Topological Transition in Josephson Junctions, Phys. Rev. Lett. 126, 036802 (2021).

[27] S. Vaitiekėnas, G. W. Winkler, B. van Heck, T. Karzig, M.-T. Deng, K. Flensberg, L. I. Glazman, C. Nayak, P. Krogstrup,
R. M. Lutchyn, and C. M. Marcus, Flux-induced topological superconductivity in full-shell nanowires, Science 367, eaav3392 (2020).

[28] T.-P. Choy, J. M. Edge, A. R. Akhmerov, and C. W. J. Beenakker, Majorana fermions emerging from magnetic nanoparticles on a superconductor without spin-orbit coupling, Phys. Rev. B 84, 195442 (2011).

[29] S. Nadj-Perge, I. K. Drozdov, B. A. Bernevig, and A. Yazdani, Majorana fermions emerging from magnetic nanoparticles on a superconductor without spin-orbit coupling, Phys. Rev. B 88, 020407(R) (2013).

[30] S. Nadj-Perge, I. K. Drozdov, J. Li, H. Chen, S. Jeon, J. Seo, A. H. MacDonald, B. A. Bernevig, and A. Yazdani, Observation of Majorana fermions in ferromagnetic atomic chains on a superconductor, Science 346, 602 (2014).

[31] B. E. Feldman, M. T. Randeria, J. Li, S. Jeon, Y. Xie, Z. Wang, I. K. Drozdov, B. A. Bernevig, and A. Yazdani, Highresolution studies of the Majorana atomic chain platform, Nat. Phys. 13, 286 (2017).

[32] G. L. Fatin, A. Matos-Abiague, B. Scharf, and I. Žutić, Wireless Majorana Bound States: From Magnetic Tunability to Braiding, Phys. Rev. Lett. 117, 077002 (2016).

[33] M. M. Desjardins, L. C. Contamin, M. R. Delbecq, M. C. Dartiailh, L. E. Bruhat, T. Cubaynes, J. J. Viennot, F. Mallet, S. Rohart, A. Thiaville, A. Cottet, and T. Kontos, Synthetic spin-orbit interaction for Majorana devices, Nat. Mater. 18, 1060 (2019).

[34] L. Fu and C. L. Kane, Superconducting Proximity Effect and Majorana Fermions at the Surface of a Topological Insulator, Phys. Rev. Lett. 100, 096407 (2008).

[35] D. Wang, L. Kong, P. Fan, H. Chen, S. Zhu, W. Liu, L. Cao, Y. Sun, S. Du, J. Schneeloch, R. Zhong, G. Gu, L. Fu, H. Ding, and H.-J. Gao, Evidence for Majorana bound states in an ironbased superconductor, Science 362, eaao1797096407 (2018).

[36] S. Sasaki, M. Kriener, K. Segawa, K. Yada, Y. Tanaka, M. Sato, and Y. Ando, Topological Superconductivity in $\mathrm{Cu}_{x} \mathrm{Bi}_{2} \mathrm{Se}_{3}$, Phys. Rev. Lett. 107, 217001 (2011).

[37] A. Yamakage, K. Yada, M. Sato, and Y. Tanaka, Theory of tunneling conductance and surface-state transition in superconducting topological insulators, Phys. Rev. B 85, 180509(R) (2012).

[38] X.-L. Qi, T. L. Hughes, and S.-C. Zhang, Chiral topological superconductor from the quantum Hall state, Phys. Rev. B 82, 184516 (2010).

[39] Q. L. He, L. Pan, A. L. Stern, E. C. Burks, X. Che, G. Yin, J. Wang, B. Lian, Q. Zhou, E. S. Choi, K. Murata, X. Kou, Z. Chen, T. Nie, Q. Shao, Y. Fan, S.-C. Zhang, K. Liu, J. Xia, and K. L. Wang, Chiral Majorana fermion modes in a quantum anomalous Hall insulator-superconductor structure, Science 357, 294 (2017).

[40] R.-J. Slager, L. Rademaker, J. Zaanen, and L. Balents, Impurity-bound states and Green's function zeros as local signatures of topology, Phys. Rev. B 92, 085126 (2015).

[41] W. A. Benalcazar, J. C. Y. Teo, and T. L. Hughes, Classification of two-dimensional topological crystalline superconductors and Majorana bound states at disclinations, Phys. Rev. B 89, 224503 (2014).

[42] Z. Song, Z. Fang, and C. Fang, $(d-2)$-Dimensional Edge States of Rotation Symmetry Protected Topological States, Phys. Rev. Lett. 119, 246402 (2017). 
[43] J. Langbehn, Y. Peng, L. Trifunovic, F. von Oppen, and P. W. Brouwer, Reflection-Symmetric Second-Order Topological Insulators and Superconductors, Phys. Rev. Lett. 119, 246401 (2017).

[44] E. Khalaf, Higher-order topological insulators and superconductors protected by inversion symmetry, Phys. Rev. B 97, 205136 (2018).

[45] Y. Wang, M. Lin, and T. L. Hughes, Weak-pairing higher order topological superconductors, Phys. Rev. B 98, 165144 (2018).

[46] Z. Yan, F. Song, and Z. Wang, Majorana Corner Modes in a High-Temperature Platform, Phys. Rev. Lett. 121, 096803 (2018).

[47] Q. Wang, C.-C. Liu, Y.-M. Lu, and F. Zhang, HighTemperature Majorana Corner States, Phys. Rev. Lett. 121, 186801 (2018).

[48] T. Liu, J. J. He, and F. Nori, Majorana corner states in a two-dimensional magnetic topological insulator on a high-temperature superconductor, Phys. Rev. B 98, 245413 (2018).

[49] M. J. Gray, J. Freudenstein, S. Y. F. Zhao, R. O'Connor, S. Jenkins, N. Kumar, M. Hoek, A. Kopec, S. Huh, T. Taniguchi, K. Watanabe, R. Zhong, C. Kim, G. D. Gu, and K. S. Burch, Evidence for helical hinge zero modes in an Fe-based superconductor, Nano Lett. 19, 4890 (2019).

[50] X. Zhu, Second-Order Topological Superconductors with Mixed Pairing, Phys. Rev. Lett. 122, 236401 (2019).

[51] R.-X. Zhang, W. S. Cole, and S. DasSarma, Helical Hinge Majorana Modes in Iron-Based Superconductors, Phys. Rev. Lett. 122, 187001 (2019).

[52] R.-X. Zhang, W. S. Cole, X. Wu, and S. DasSarma, HigherOrder Topology and Nodal Topological Superconductivity in $\mathrm{Fe}(\mathrm{Se}, \mathrm{Te})$ Heterostructures, Phys. Rev. Lett. 123, 167001 (2019).

[53] Z. Yan, Higher-Order Topological Odd-Parity Superconductors, Phys. Rev. Lett. 123, 177001 (2019).

[54] Y.-J. Wu, J. Hou, Y.-M. Li, X.-W. Luo, X. Shi, and C. Zhang, In-Plane Zeeman-Field-Induced Majorana Corner and Hinge Modes in an $s$-Wave Superconductor Heterostructure, Phys. Rev. Lett. 124, 227001 (2020).

[55] M. Kheirkhah, Z. Yan, Y. Nagai, and F. Marsiglio, First- and Second-Order Topological Superconductivity and Temperature-Driven Topological Phase Transitions in the Extended Hubbard Model with Spin-Orbit Coupling, Phys. Rev. Lett. 125, 017001 (2020).

[56] Y.-T. Hsu, W. S. Cole, R.-X. Zhang, and J. D. Sau, InversionProtected Higher-Order Topological Superconductivity in Monolayer $\mathrm{WTe}_{2}$, Phys. Rev. Lett. 125, 097001 (2020).

[57] K. Plekhanov, N. Müller, Y. Volpez, D. M. Kennes, H. Schoeller, D. Loss, and J. Klinovaja, Quadrupole spin polarization as signature of second-order topological superconductors, Phys. Rev. B 103, L041401 (2021).

[58] G. E. Volovik, Topological superfluid ${ }^{3} \mathrm{He}-\mathrm{B}$ in magnetic field and Ising variable, JETP Lett. 91, 201 (2010).

[59] X. Zhu, Tunable Majorana corner states in a two-dimensional second-order topological superconductor induced by magnetic fields, Phys. Rev. B 97, 205134 (2018).

[60] Y. Volpez, D. Loss, and J. Klinovaja, Second-Order Topological Superconductivity in $\pi$-Junction Rashba Layers, Phys. Rev. Lett. 122, 126402 (2019).
[61] K. Laubscher, D. Loss, and J. Klinovaja, Fractional topological superconductivity and parafermion corner states, Phys. Rev. Research 1, 032017(R) (2019).

[62] X. Wu, X. Liu, R. Thomale, and C.-X. Liu, High- $T_{c}$ superconductor $\mathrm{Fe}(\mathrm{Se}, \mathrm{Te})$ monolayer: An intrinsic, scalable and electrically-tunable Majorana platform, arXiv:1905.10648.

[63] S.-B. Zhang, W. B. Rui, A. Calzona, S.-J. Choi, A. P. Schnyder, and B. Trauzettel, Topological and holonomic quantum computation based on second-order topological superconductors, Phys. Rev. Research 2, 043025 (2020).

[64] T. E. Pahomi, M. Sigrist, and A. A. Soluyanov, Braiding Majorana corner modes in a second-order topological superconductor, Phys. Rev. Research 2, 032068(R) (2020).

[65] S.-B. Zhang, A. Calzona, and B. Trauzettel, All-electrically tunable networks of Majorana bound states, Phys. Rev. B 102, 100503(R) (2020).

[66] S. Fujimoto, Electron correlation and pairing states in superconductors without inversion symmetry, J. Phys. Soc. Jpn. 76, 051008 (2007).

[67] M. Smidman, M. B. Salamon, H. Q. Yuan, and D. F. Agterberg, Superconductivity and spin-orbit coupling in noncentrosymmetric materials: A review, Rep. Prog. Phys. 80, 036501 (2017).

[68] C. Iniotakis, N. Hayashi, Y. Sawa, T. Yokoyama, U. May, Y. Tanaka, and M. Sigrist, Andreev bound states and tunneling characteristics of a noncentrosymmetric superconductor, Phys. Rev. B 76, 012501 (2007).

[69] Y. Tanaka, T. Yokoyama, A. V. Balatsky, and N. Nagaosa, Theory of topological spin current in noncentrosymmetric superconductors, Phys. Rev. B 79, 060505(R) (2009).

[70] M. Sato and S. Fujimoto, Topological phases of noncentrosymmetric superconductors: Edge states, Majorana fermions, and non-Abelian statistics, Phys. Rev. B 79, 094504 (2009).

[71] Y. Tanaka, Y. Mizuno, T. Yokoyama, K. Yada, and M. Sato, Anomalous Andreev Bound State in Noncentrosymmetric Superconductors, Phys. Rev. Lett. 105, 097002 (2010).

[72] A. P. Schnyder and P. M. Brydon, Topological surface states in nodal superconductors, J. Phys.: Condens. Matter 27, 243201 (2015).

[73] T. Yasuda, H. Shishido, T. Ueda, S. Hashimoto, R. Settai, T. Takeuchi, T. D. Matsuda, Y. Haga, and Y. Ōnuki, Superconducting property in $\mathrm{CePt}_{3} \mathrm{Si}$ under pressure, J. Phys. Soc. Jpn. 73, 1657 (2004).

[74] M. Yogi, H. Mukuda, Y. Kitaoka, S. Hashimoto, T. Yasuda, R. Settai, T. D. Matsuda, Y. Haga, Y. Ōnuki, P. Rogl, and E. Bauer, Evidence for novel pairing state in noncentrosymmetric superconductor $\mathrm{CePt}_{3} \mathrm{Si}$ : ${ }^{29} \mathrm{Si}-\mathrm{NMR}$ Knight shift study, J. Phys. Soc. Jpn. 75, 013709 (2006).

[75] E. Bauer, G. Hilscher, H. Michor, C. Paul, E. W. Scheidt, A. Gribanov, Y. Seropegin, H. Noël, M. Sigrist, and P. Rogl, Heavy Fermion Superconductivity and Magnetic Order in Noncentrosymmetric $\mathrm{CePt}_{3} \mathrm{Si}$, Phys. Rev. Lett. 92, 027003 (2004).

[76] K. Izawa, Y. Kasahara, Y. Matsuda, K. Behnia, T. Yasuda, R. Settai, and Y. Onuki, Line Nodes in the Superconducting Gap Function of Noncentrosymmetric $\mathrm{CePt}_{3} \mathrm{Si}$, Phys. Rev. Lett. 94, 197002 (2005).

[77] I. Bonalde, W. Brämer-Escamilla, and E. Bauer, Evidence for Line Nodes in the Superconducting Energy Gap of 
Noncentrosymmetric $\mathrm{CePt}_{3} \mathrm{Si}$ from Magnetic Penetration Depth Measurements, Phys. Rev. Lett. 94, 207002 (2005).

[78] H. Mukuda, S. Nishide, A. Harada, K. Iwasaki, M. Yogi, M. Yamashita, Y. Kitaoka, M. Tsujino, T. Takeuchi, R. Settai, Y. Ōnuki, E. Bauer, K. M. Itoh, and E. E. Haller, Multiband superconductivity in heavy fermion compound $\mathrm{CePt}_{3} \mathrm{Si}$ without inversion symmetry: An NMR study on a high-quality single crystal, J. Phys. Soc. Jpn. 78, 014705 (2009).

[79] P. A. Frigeri, D. F. Agterberg, A. Koga, and M. Sigrist, Superconductivity without Inversion Symmetry: MnSi versus $\mathrm{CePr}_{3} \mathrm{Si}$, Phys. Rev. Lett. 92, 097001 (2004).

[80] S. Fujimoto, Magnetoelectric effects in heavy-fermion superconductors without inversion symmetry, Phys. Rev. B 72, 024515 (2005).

[81] N. Hayashi, K. Wakabayashi, P. A. Frigeri, and M. Sigrist, Nuclear magnetic relaxation rate in a noncentrosymmetric superconductor, Phys. Rev. B 73, 092508 (2006).

[82] Y. Yanase, and M. Sigrist, Superconductivity and magnetism in non-centrosymmetric system: Application to $\mathrm{CePt}_{3} \mathrm{Si}$, J. Phys. Soc. Jpn. 77, 124711 (2008).

[83] A. P. Schnyder, S. Ryu, A. Furusaki, and A. W. W. Ludwig, Classification of topological insulators and superconductors in three spatial dimensions, Phys. Rev. B 78, 195125 (2008).

[84] X.-L. Qi, T. L. Hughes, S. Raghu, and S.-C. Zhang, TimeReversal-Invariant Topological Superconductors and Superfluids in Two and Three Dimensions, Phys. Rev. Lett. 102, 187001 (2009).

[85] C. L. M. Wong, J. Liu, K. T. Law, and P. A. Lee, Majorana flat bands and unidirectional Majorana edge states in gapless topological superconductors, Phys. Rev. B 88, 060504(R) (2013).

[86] P. A. Lee and D. S. Fisher, Anderson Localization in Two Dimensions, Phys. Rev. Lett. 47, 882 (1981).

[87] S. B. Chung and S.-C. Zhang, Detecting the Majorana Fermion Surface State of ${ }^{3} \mathrm{He}-\mathrm{B}$ through Spin Relaxation, Phys. Rev. Lett. 103, 235301 (2009).

[88] R. Shindou, A. Furusaki, and N. Nagaosa, Quantum impurity spin in Majorana edge fermions, Phys. Rev. B 82, 180505(R) (2010).
[89] Y. Xiong, A. Yamakage, S. Kobayashi, M. Sato, and Y. Tanaka, Anisotropic magnetic responses of topological crystalline superconductors, Crystals 7, 58 (2017).

[90] A. C. Potter and P. A. Lee, Multichannel Generalization of Kitaev's Majorana End States and a Practical Route to Realize Them in Thin Films, Phys. Rev. Lett. 105, 227003 (2010)

[91] M. Wimmer, A. R. Akhmerov, and F. Guinea, Robustness of edge states in graphene quantum dots, Phys. Rev. B 82, 045409 (2010).

[92] R. Queiroz and A. P. Schnyder, Stability of flat-band edge states in topological superconductors without inversion center, Phys. Rev. B 89, 054501 (2014).

[93] G. E. Blonder, M. Tinkham, and T. M. Klapwijk, Transition from metallic to tunneling regimes in superconducting microconstrictions: Excess current, charge imbalance, and supercurrent conversion, Phys. Rev. B 25, 4515 (1982).

[94] C. Bruder, Andreev scattering in anisotropic superconductors, Phys. Rev. B 41, 4017 (1990).

[95] S. Kashiwaya and Y. Tanaka, Tunnelling effects on surface bound states in unconventional superconductors, Rep. Prog. Phys. 63, 1641 (2000).

[96] T. Ando, Quantum point contacts in magnetic fields, Phys. Rev. B 44, 8017 (1991).

[97] L. J. Buchholtz and G. Zwicknagl, Identification of $p$-wave superconductors, Phys. Rev. B 23, 5788 (1981).

[98] C.-R. Hu, Midgap Surface States as a Novel Signature for $d_{x_{a}^{2}-x_{b}^{2}}$-Wave Superconductivity, Phys. Rev. Lett. 72, 1526 (1994).

[99] Y. Tanaka and S. Kashiwaya, Theory of Tunneling Spectroscopy of $d$-Wave Superconductors, Phys. Rev. Lett. 74, 3451 (1995).

[100] K. Sengupta, I. Žutić, H.-J. Kwon, V. M. Yakovenko, and S. Das Sarma, Midgap edge states and pairing symmetry of quasi-one-dimensional organic superconductors, Phys. Rev. B 63, 144531 (2001).

[101] Y. Asano, Y. Tanaka, and S. Kashiwaya, Phenomenological theory of zero-energy Andreev resonant states, Phys. Rev. B 69, 134501 (2004). 